\title{
Quality of life in atopic dermatitis in Asian countries: a systematic review
}

\author{
Jinghui Huang ${ }^{1} \cdot$ Yue Jia Choo ${ }^{1} \cdot$ Helen Elizabeth Smith ${ }^{1} \cdot$ Christian Apfelbacher $^{1,2}$ (1)
}

Received: 12 May 2021 / Accepted: 22 May 2021 / Published online: 4 June 2021

(c) The Author(s) 2021

\begin{abstract}
Atopic dermatitis (AD) is a common chronic inflammatory skin condition which impacts psychological wellbeing and social relationships. There have been studies of AD's impact on quality of life (QoL) in Western countries, but these findings cannot be directly extrapolated to Asian populations with genetic, environmental and cultural differences. Therefore, we aimed to systematically review the literature pertaining to QoL impairment in AD in East and Southeast Asia to characterize the impact of $\mathrm{AD}$ on patients and their families, and to identify the factors affecting the degree of QoL impairment. A search of English language papers was conducted on MEDLINE, EMBASE, PSYCInfo, Global Health and Web of Science. Observational studies measuring QoL using single or multi-item instruments in people with self-reported or physician diagnosed atopic dermatitis were included. 27 studies from 29 articles were included and synthesized. There is data documenting QoL impairment in AD sufferers and their families, across a wide range of Asian countries, healthcare settings and ages. Aspects of QoL impacted to a greater extent included symptoms of itch, feelings of embarrassment, and sleep disturbance. Severity of disease affects the degree of impairment of QoL, but there is no apparent link between QoL impairment and patient demographic factors, or other medical factors such as age at diagnosis or duration of illness. Our findings also highlighted the need for clinicians to actively explore the impact of patient's symptoms, especially in an Asian context where healthcare communications are traditionally doctor-centric.
\end{abstract}

Keyword Atopic dermatitis · Quality of life · Asian · Systematic review

$\begin{array}{ll}\text { Abbreviations } \\ \text { AD } & \text { Atopic dermatitis } \\ \text { QoL } & \text { Quality of life } \\ \text { HC } & \text { Healthy controls } \\ \text { CDLQI } & \text { Children's Dermatology Life Quality Index } \\ \text { DLQI } & \text { Dermatology Life Quality Index } \\ \text { PCS } & \text { Physical Component Scores } \\ \text { MCS } & \text { Mental Component Scores } \\ \text { DFI } & \text { Dermatitis Family Impact } \\ \text { POEM } & \text { Patient-Oriented Eczema Measure } \\ \text { NESS } & \text { Nottingham Eczema Severity Score } \\ \text { SCORAD } & \text { SCORing Atopic Dermatitis }\end{array}$

Christian Apfelbacher

christian.apfelbacher@med.ovgu.de

1 Department of Family Medicine and Primary Care, Nanyang Technological University, Lee Kong Chian School of Medicine, Singapore, Singapore

2 Institute of Social Medicine and Health Systems Research, Otto Von Guericke University Magdeburg, Leipziger Str. 44, 39120 Magdeburg, Germany

$\begin{array}{ll}\text { SD } & \text { Standard deviation } \\ \text { NR } & \text { Not reported } \\ \text { SF } & \text { Short Form } \\ \text { yo } & \text { Years-old }\end{array}$

\section{Introduction}

Atopic dermatitis (AD) is a common type of chronic inflammatory skin condition, with a particular subtype associated with a heightened immune response to common allergens [1]. It is characterized by itchy lesions most commonly on the flexural surfaces and affects mainly children.

Quality of life (QoL) is a multidimensional construct encompassing one's mental, physical and social wellbeing. While non-life threatening, atopic dermatitis is associated with QoL impairment; the itching may affect mood and sleep hygiene, and the lesions may cause embarrassment, thus impacting on psychological wellbeing and social relationships [1]. The wellbeing of carers may also be affected, as they often must modify their lifestyle to provide care. In 
view of its importance, QoL has been included as one of the four core outcome domains to ideally be measured in all atopic dermatitis clinical trials [2]. While QoL of AD patients has been widely studied in Western countries [3-5], these findings cannot automatically be extrapolated to the Asian context as genetic, environmental and cultural factors may affect clinical manifestation and prevalence of $\mathrm{AD}$ between races $[6,7]$. It is has been reported that filaggrin null mutations which vary between people of different ethnicities may be positively correlated with the severity of $\mathrm{AD}$ [8]. For example, the Filaggrin null mutation c.3321delA is not found in western populations but has been reported in East Asian populations such as Japan, China, Korea, Taiwan and Singapore [6]. Differences in the cultural context may also lead to differences in individual's perception of their disease. In addition the greater involvement of family with the Confucian principle of family-centered care giving [9], may impact on family QoL.

With these differences, it was pertinent to summarize what is known about the impact of AD specifically in the Asian context. Hence, this systematic review aimed to qualitatively synthesize and critique the published literature with two research questions in mind. First, does atopic dermatitis impact QoL in Asian countries, and if so, how and to what extent? Second, what are the determinants of QoL in atopic dermatitis among the Asian population?

\section{Methods}

The protocol was first developed using the PRISMA Statement [10] as a guideline. Details of the protocol were registered on PROSPERO and can be accessed at http://www. crd.york.ac.uk/PROSPERO/display_record.php?ID=CRD42 018106613.

A search strategy was developed with MeSH headings and keywords relevant to the population (people with atopic dermatitis), study design (observational studies), context (people from Asian countries), and outcome (QoL). The search for relevant studies was conducted in Aug 2018 on electronic databases MEDLINE, EMBASE, PSYCInfo, Global Health, and Web of Science (from inception to 21 Aug 2018).

The review process was conducted independently by two of our authors (Huang and Choo). Titles and abstracts of studies were retrieved and screened for their relevance to the research question using the inclusion/exclusion criteria in Table 1. Potentially eligible articles were then accessed, and their full text assessed for eligibility. Full-text articles failing to fulfil the selection criteria were excluded and the reasons documented.

Data extraction were then conducted on full text articles which were eligible, using a pre-piloted form. Data extracted included general information, sample characteristics, study methodology, outcome measurements, and any other significant results or factors not in our pre-determined categories. The form was continuously reviewed and modified to reflect more accurately the information included in studies.

\section{Data analysis}

A qualitative synthesis of the findings was carried out and reported as a narrative summary.

Additional analysis was conducted on the Children's Dermatology Life Quality Index (CDLQI) and Dermatology Life Quality Index (DLQI) scores. The CDLQI and DLQI scores were interpreted using validated severity stratifications by Waters et al. [11] and Hongbo et al. [12], respectively, to determine the degree of QoL impairment. Scores for each question in these questionnaires were grouped under six headings, and an aggregate score for each heading was calculated as according to the CDLQI and DLQI instructions for detailed analysis. The aspects of QoL with the three highest scores from each study were then highlighted to identify trends.

\section{Risk of bias assessment}

Included studies were assessed by both reviewers for risk of selection bias and information bias using an adapted tool

Table 1 Inclusion/exclusion criteria

\begin{tabular}{|c|c|}
\hline Aspect of study & Criteria \\
\hline Population & $\begin{array}{l}\text { Included: people of any age group diagnosed with atopic dermatitis using any diagnostic criteria or self-reported } \\
\text { diagnoses. Both population-based studies and studies using clinical samples were included }\end{array}$ \\
\hline Study type & $\begin{array}{l}\text { Included: observational studies } \\
\text { Excluded: interventional studies, case reports, case series and studies which do not report primary data }\end{array}$ \\
\hline Context & $\begin{array}{l}\text { Included: studies of populations in East Asia or Southeast Asia } \\
\text { Excluded: ethnic Asians living outside of Asia }\end{array}$ \\
\hline Outcome & Included: studies which used single or multi-item instruments measuring QoL involving self or proxy reported data \\
\hline Language & Included: English language papers only \\
\hline Type of article & Included: journal articles only \\
\hline
\end{tabular}


(Appendix) based on Dodoo-Schittko et al. [13], and modified from the Newcastle-Ottawa Scale.

\section{Results}

The search yielded 4396 articles after removal of the duplicates. After screening and assessing full text for eligibility, 27 studies from 29 articles were included, as outlined in the PRISMA flow diagram (Fig. 1). The extracted data from the included studies are represented below (Table 2). Of the 27 studies, the countries represented included Singapore (four studies), Malaysia (two studies), Japan (five studies), Korea (six studies), Hong Kong (nine studies) and Taiwan (one study). Most studies were performed on patients under specialist care (dermatology clinics or hospitals), only one study was conducted in a primary care setting [14]. Four studies were population surveys using self-reported diagnoses to identify people with AD [15-19]. Nine QoL instruments were used, with the most used being Infant Dermatitis Quality of Life Index (four studies), CDLQI (15 studies), DLQI (six studies), Short form Health survey (four studies) and Euro-QoL-5 Dimension Index (three studies).

\section{QoL impairment in people with atopic dermatitis}

QoL was impaired in Asian people with AD. Mean IDQoL scores ranged from 6.8 to 7.7 , but there is no validated interpretation for the absolute value of IDQoL [20]. CDLQI and DLQI scores ranged for 4.8-15.2 and 4.8-12.0, respectively, with most studies describing a "moderate" or "very large effect" on QoL as according to the interpretation by Waters et al. [11] and Hongbo et al. [12].

The Short Form Health Survey indicated that AD patients have a statistically significant QoL impairment in both Physical Component Scores (PCS) and Mental Component Scores (MCS) compared to healthy controls (HCs) $[15,21]$, except for one study [22] which showed only impairment in MCS and not PCS. Impairment of QoL was also statistically significant in AD patients compared to healthy controls in two studies using the EQ5D scale [16, $18,19]$, with one study [23] reporting a 30-35\% decrease in QoL by AD. Some studies showed a statistically significant QoL impairment in AD patients using Skindex-16 [24] or WHOQoL-26 [25].

Fig. 1 PRISMA flow diagram

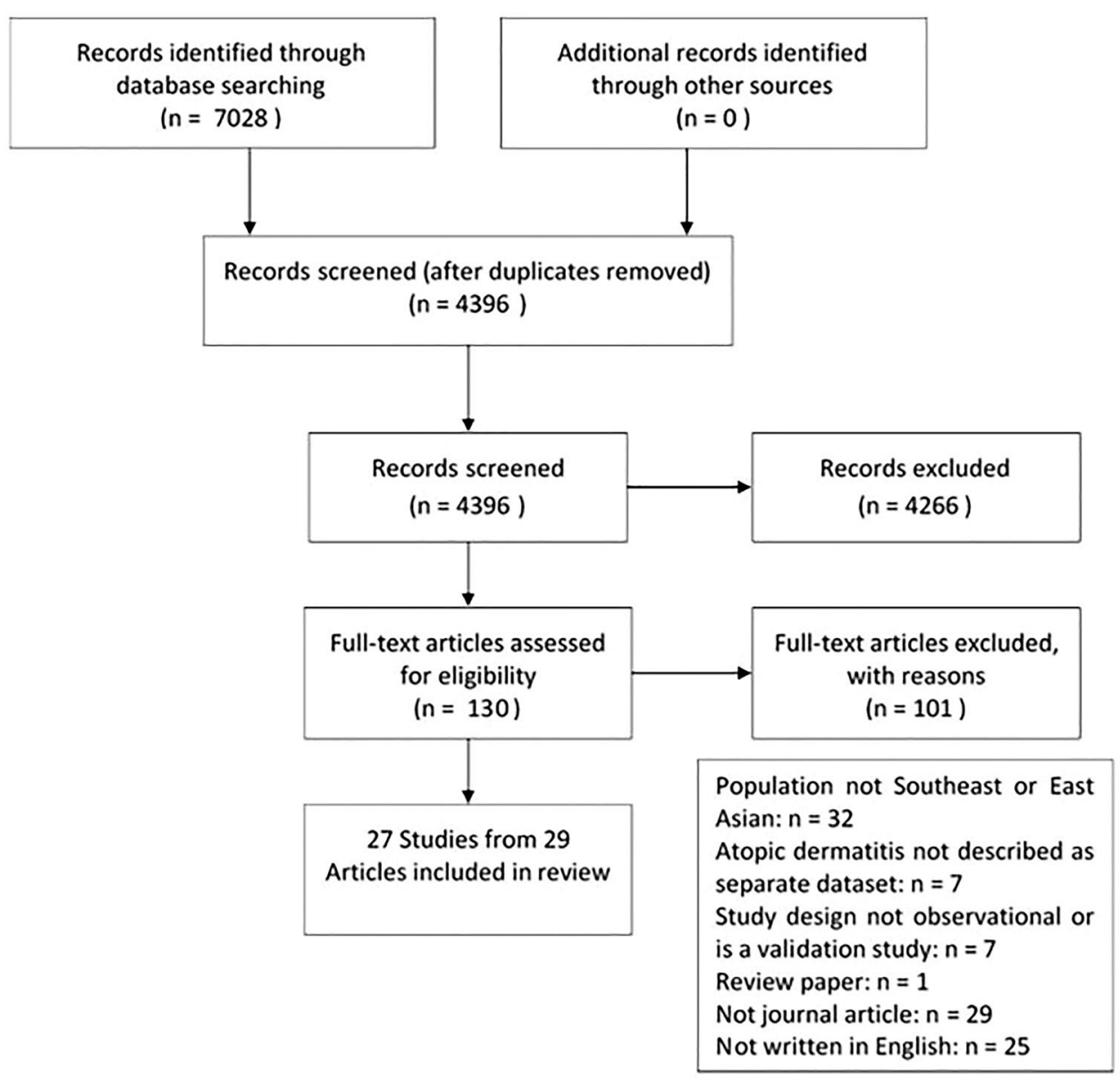




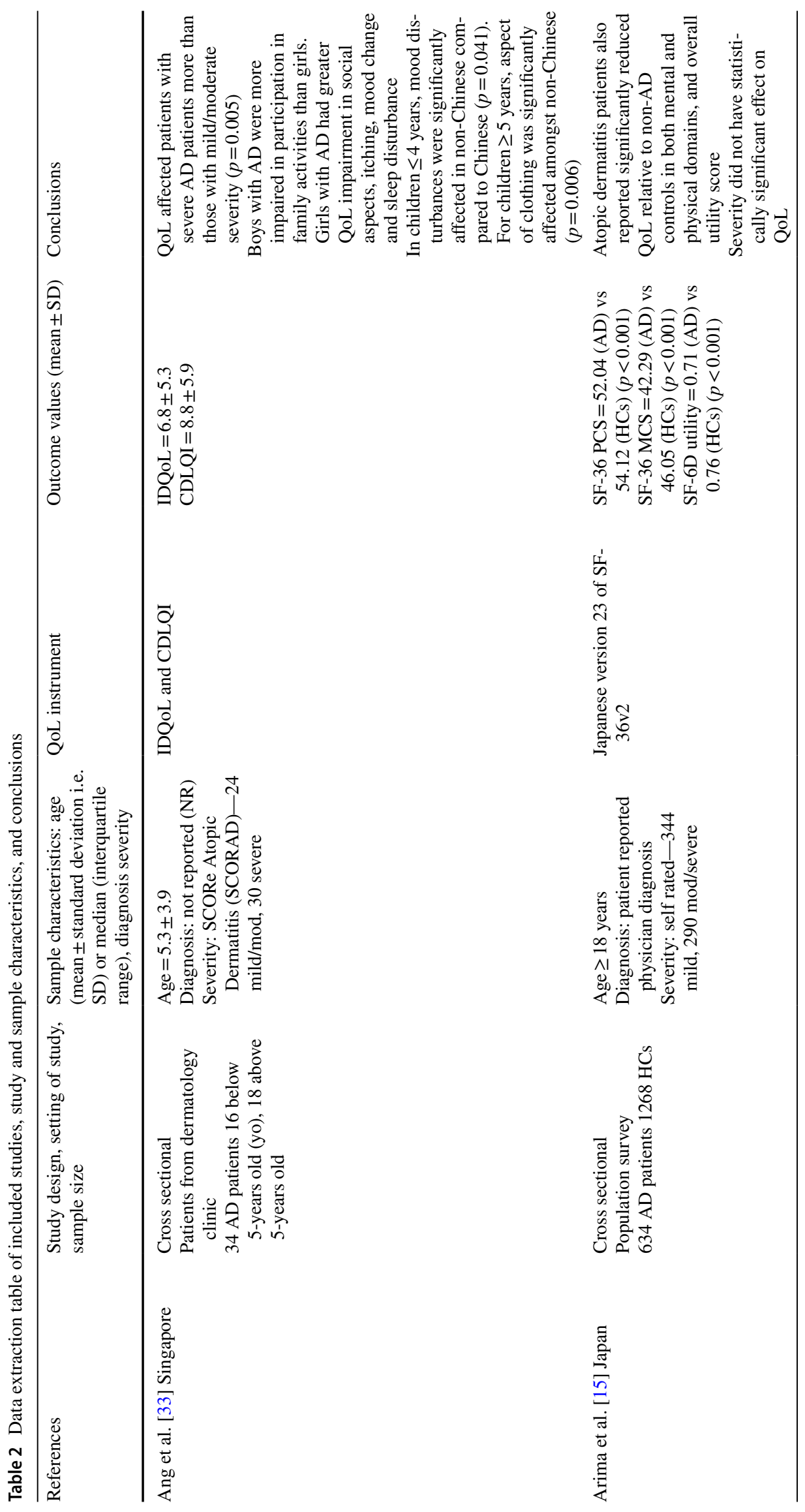




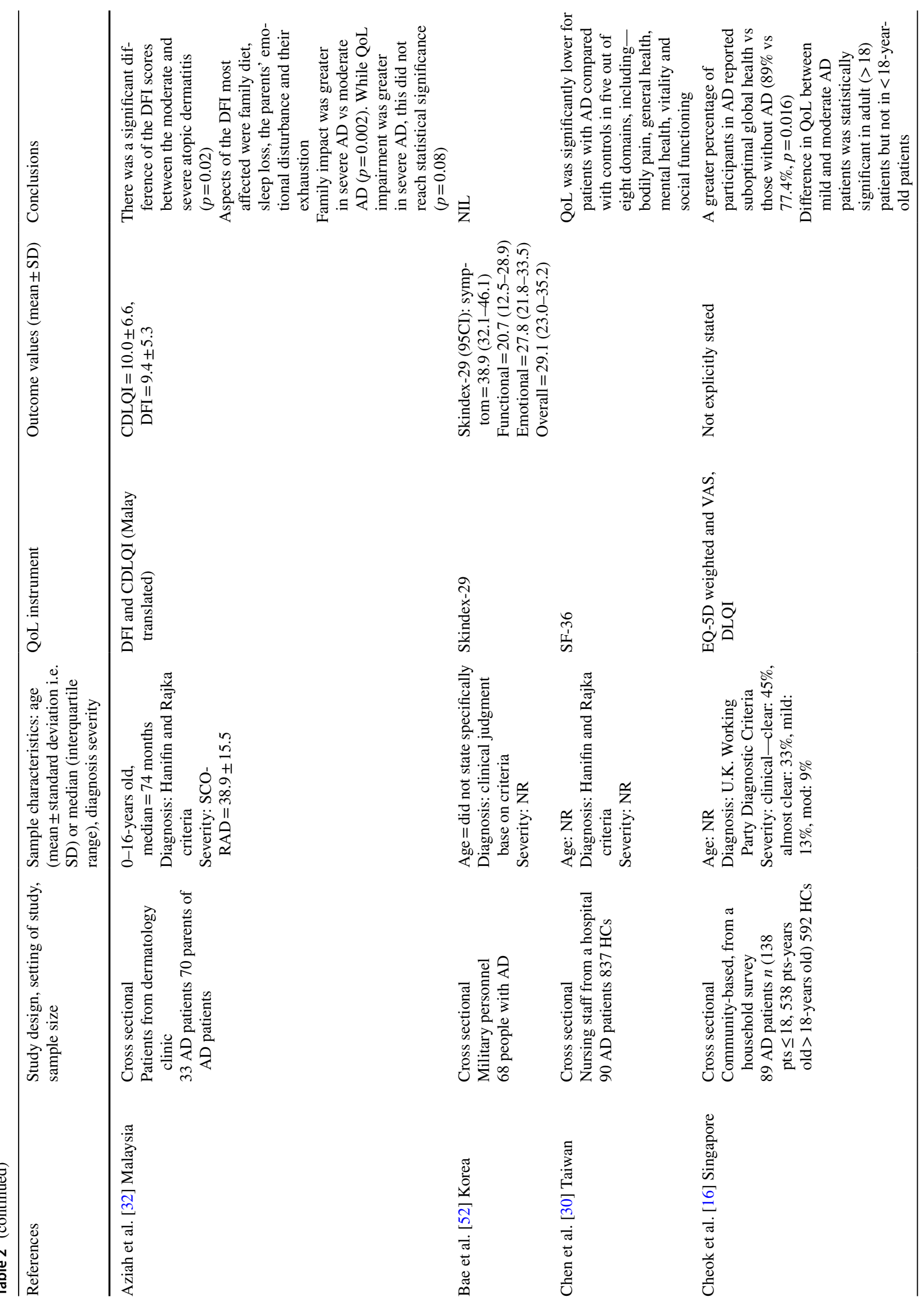




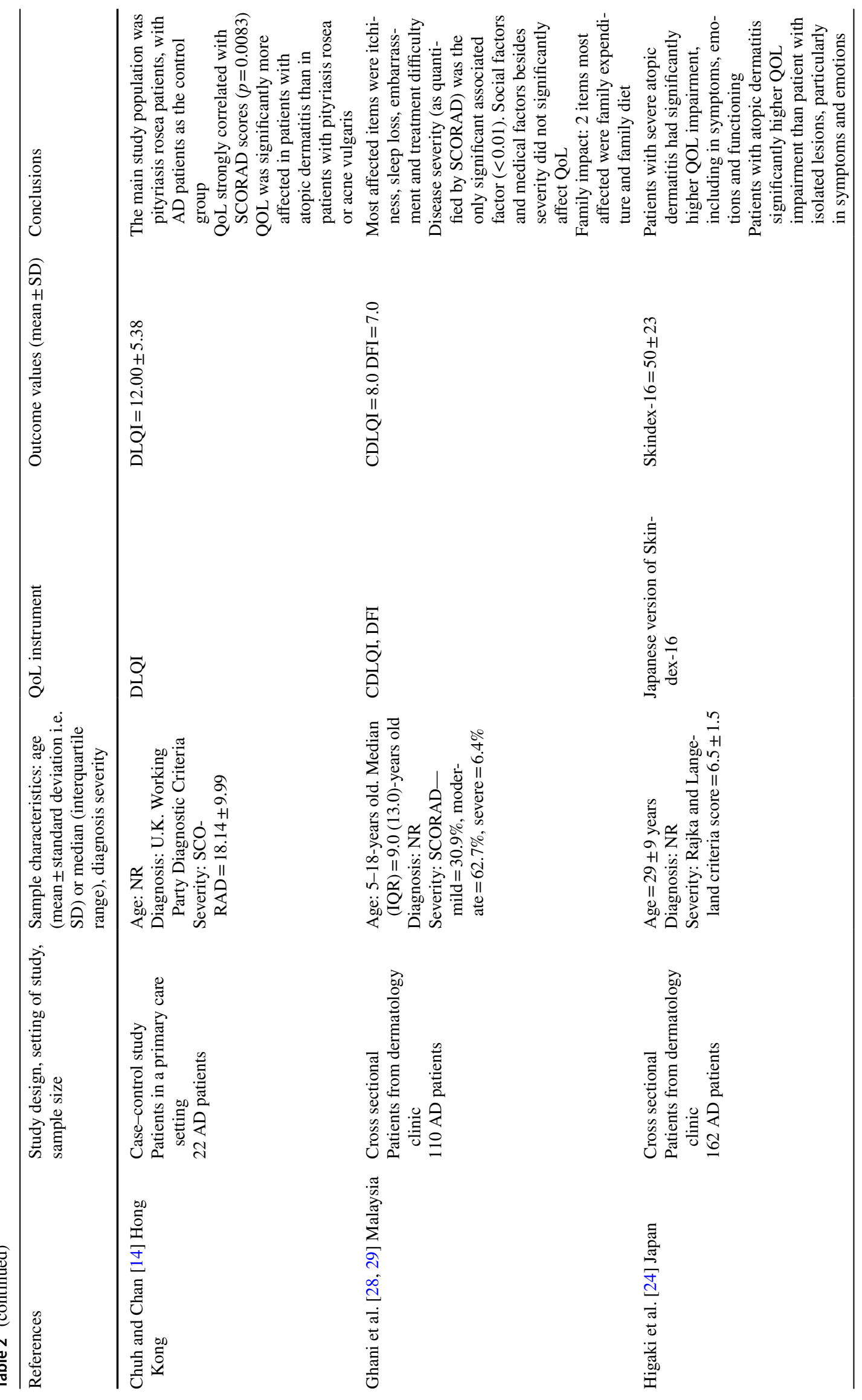




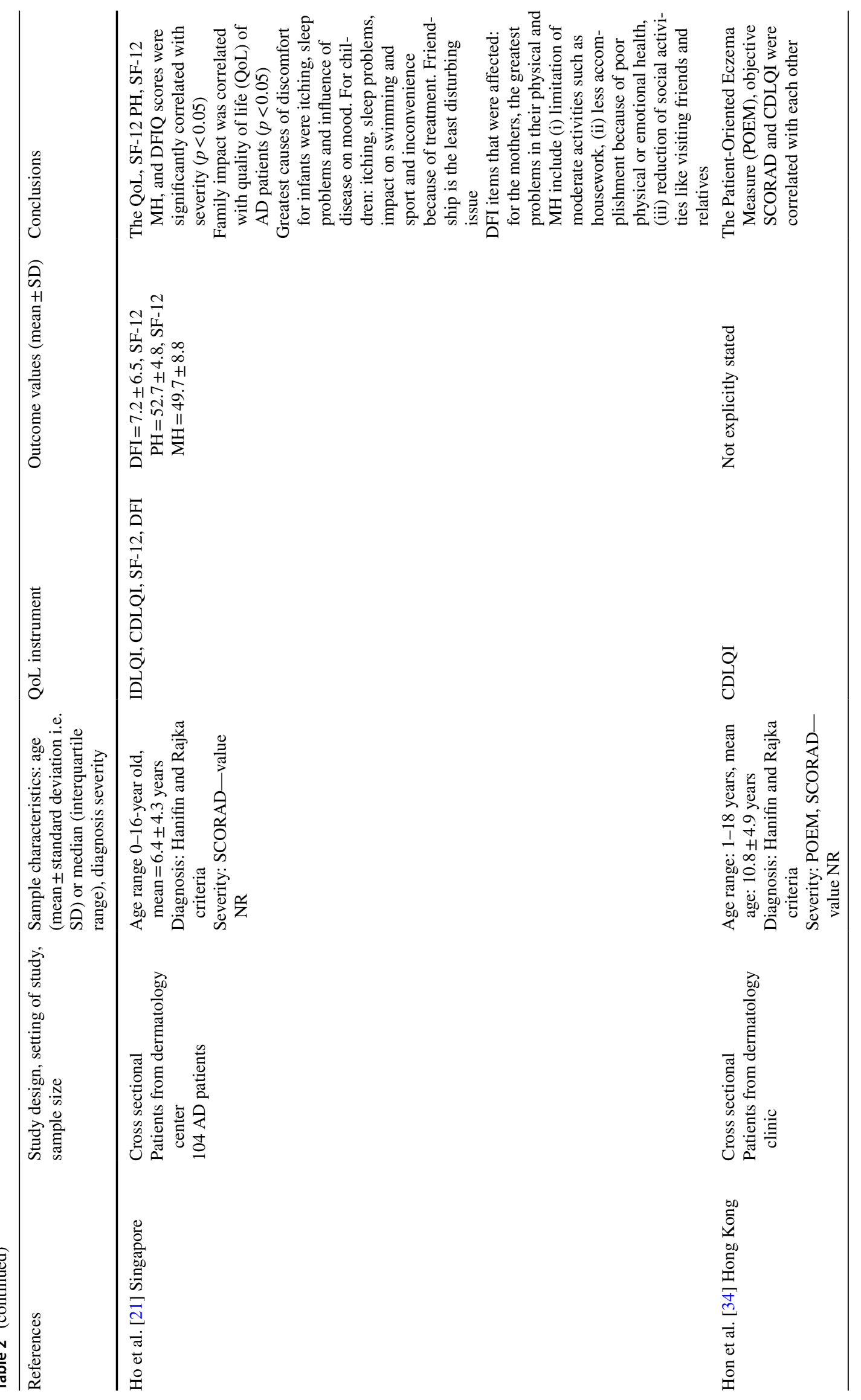




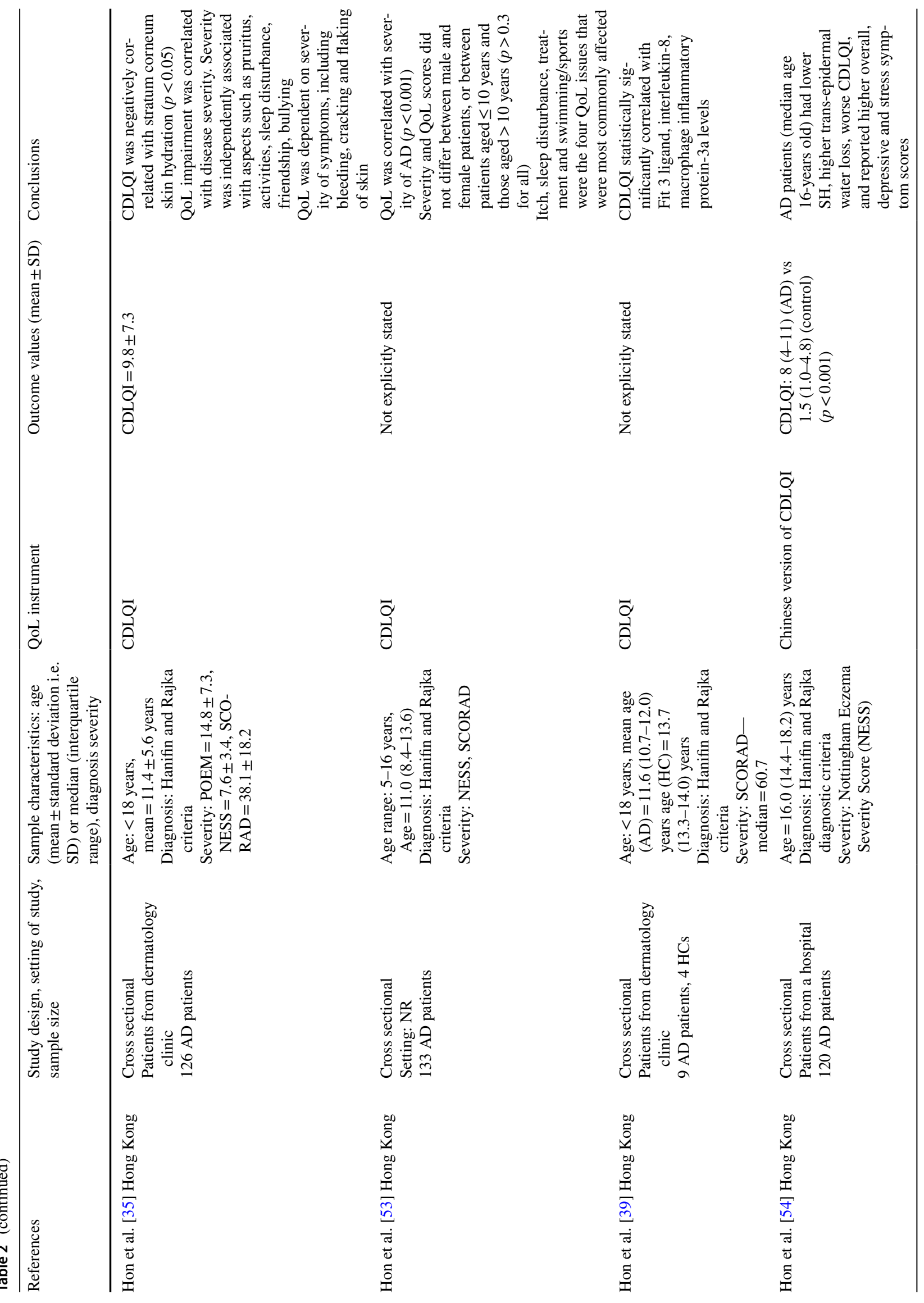




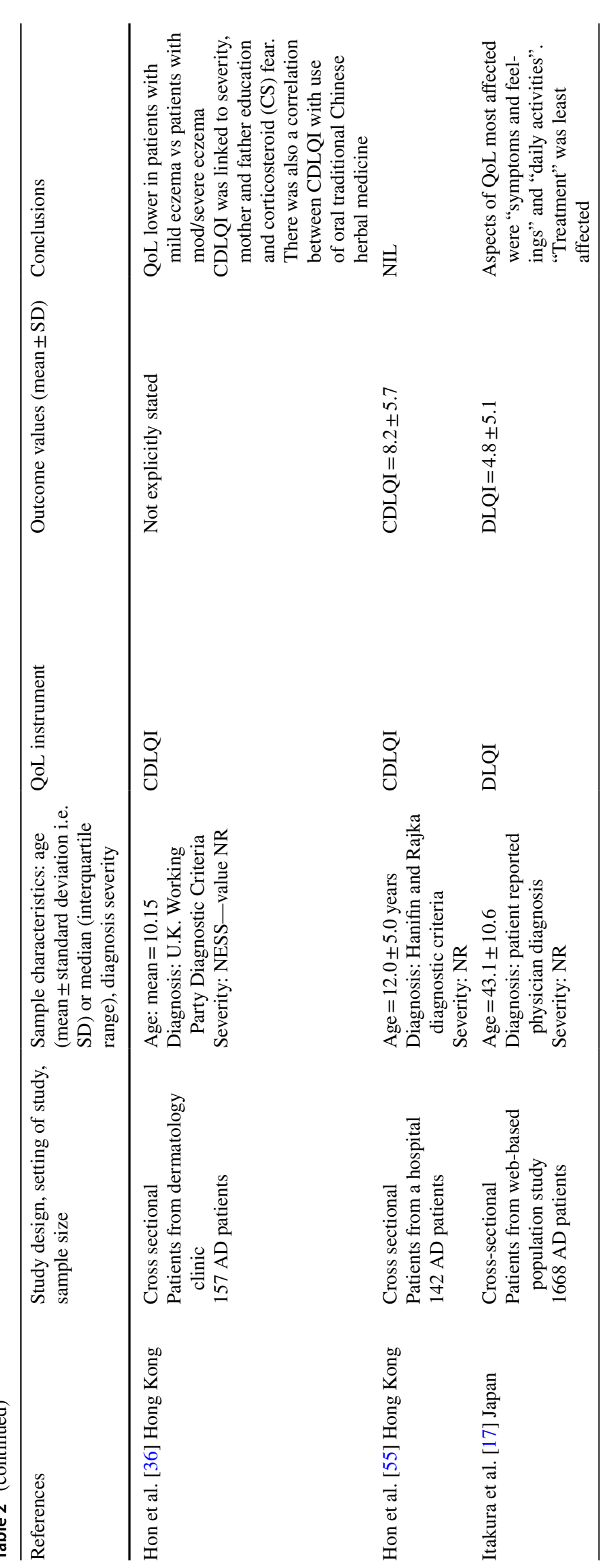




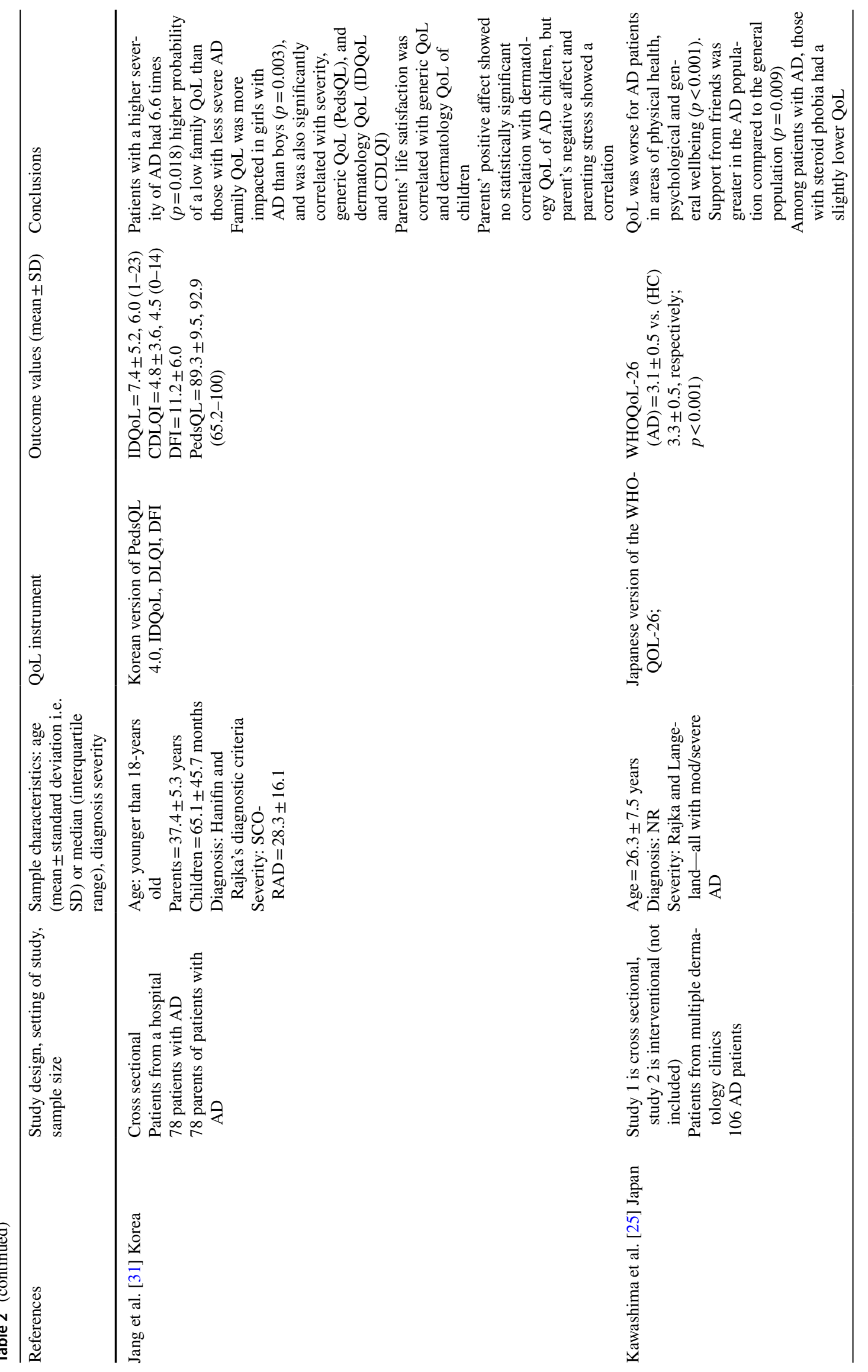




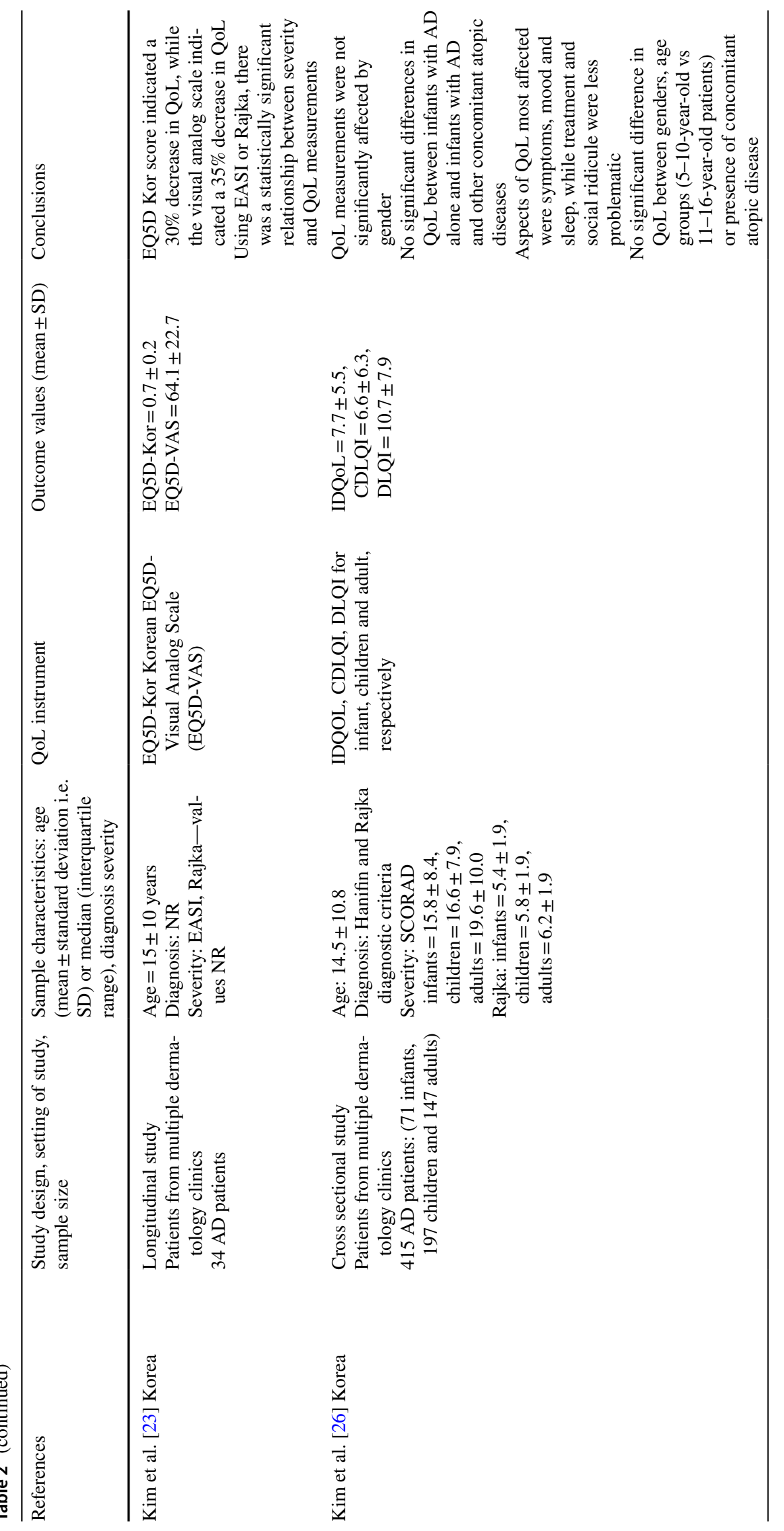




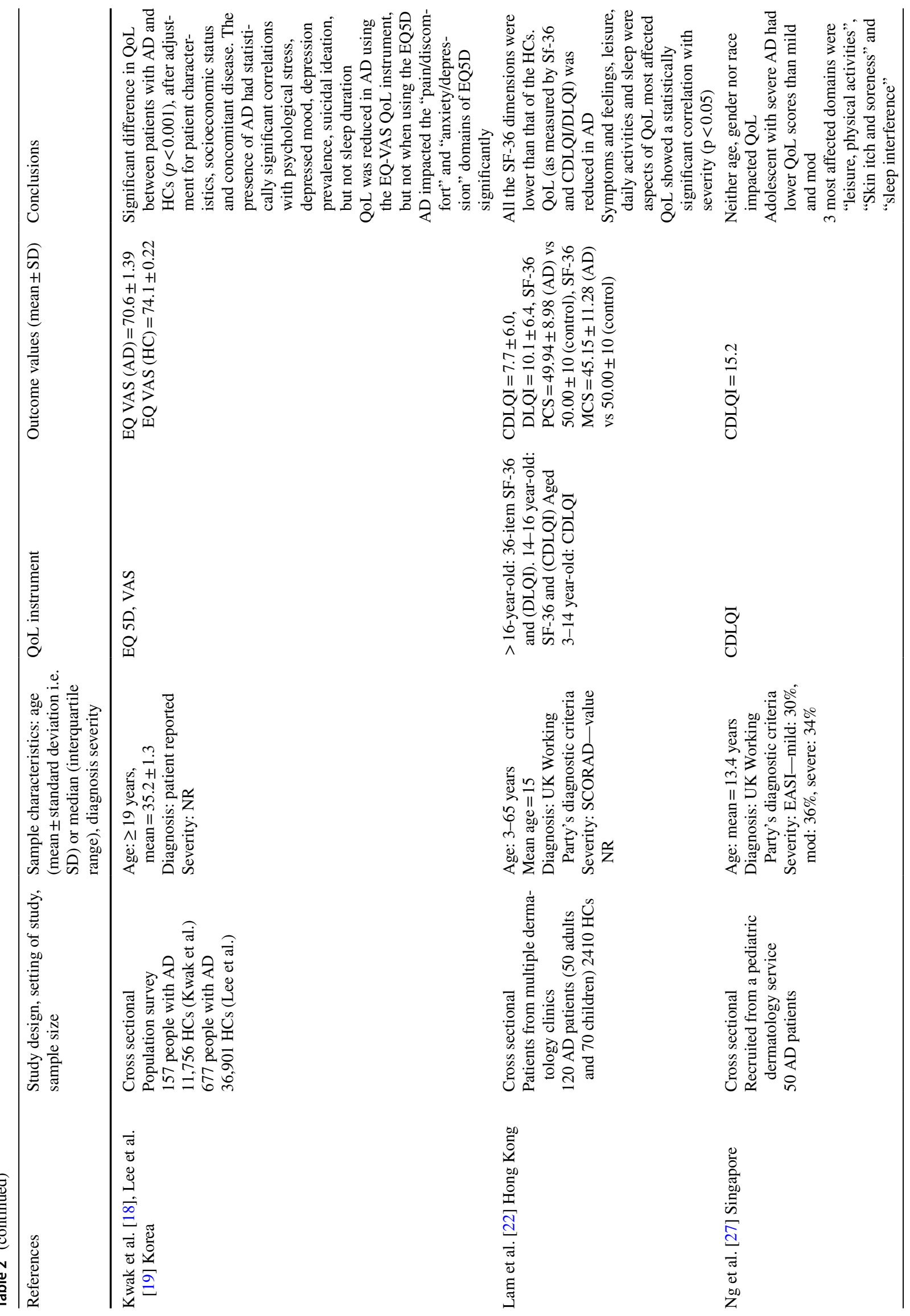




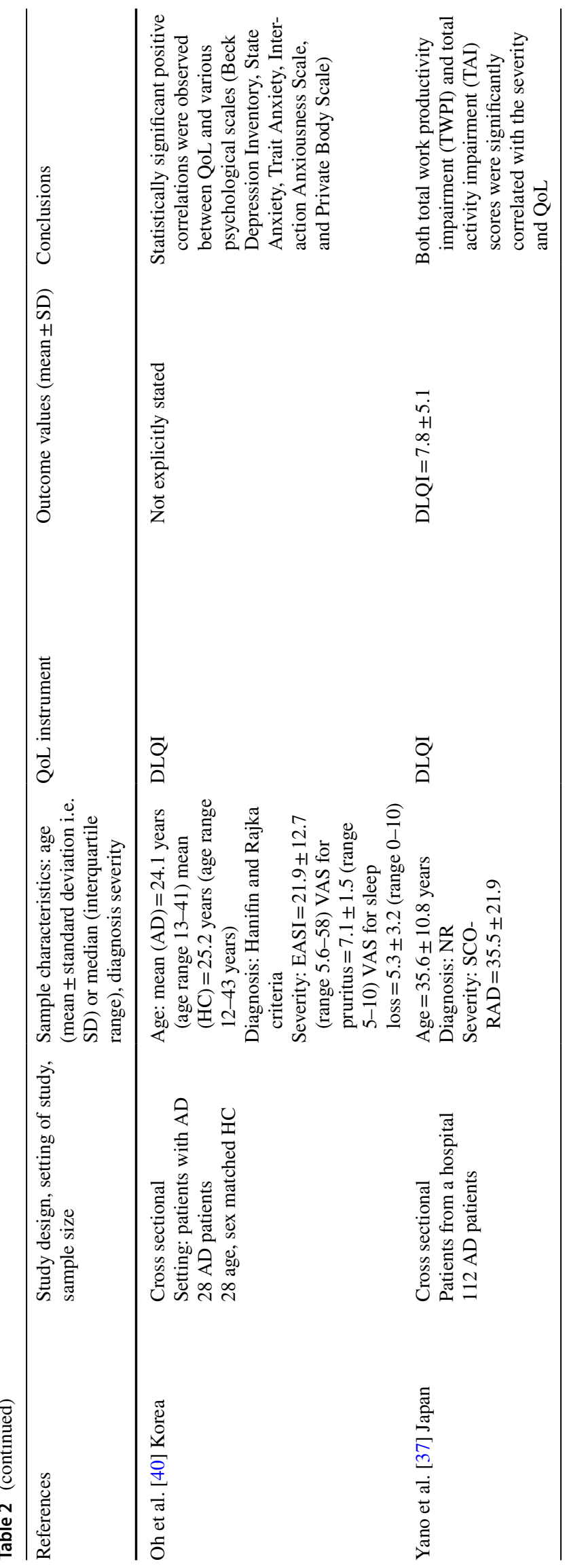

\section{Aspects of QoL affected are in the domains of symptoms and feelings, and sleep}

The aspects of QoL affected were specified numerically in only four studies using CDLQI and four studies using DLQI (Table 3), while other studies using these QoL instruments only described the various aspects of QoL affected qualitatively. Symptoms of itch and feelings of embarrassment were the most pertinent aspects of QoL. Furthermore, sleep appeared to be an important aspect of QoL [22, 26-29], though one study $[18,19]$ showed no difference in sleep duration between AD patients and healthy controls. The findings from the analysis of CDLQI and DLQI was corroborated by Higaki et al. [24], which found that "symptoms" and "feelings" were more impacted than "functioning". One study [30] using Short Form (SF)-36 and one [18, 19] using EQ5D also showed similar results, with statistically significant impact in aspects of QoL such as symptoms of "pain" and "discomfort", and mental wellbeing.

\section{QoL impairment in family members of people with AD}

AD also impacts the QoL of family members. The Dermatitis Family Impact (DFI) scores ranged from 4.8 to 9.4 [21, 28, 29, 31]. Applying the interpretation proposed by Ricci et al. [3], three studies [21, 28, 29, 32] showed a minor impact, while one study [31] showed no impact in QoL.

In particular, the aspects most affected were family diet [28, 29, 32] and emotional wellbeing [21, 32]. Limitations to social and personal activities [21], sleep loss [32] and expenditure $[28,29]$ also were documented.

\section{Determinants of QoL in people with AD}

Of the 15 studies investigating the relationship between severity and QoL in AD, 14 showed a statistically significant correlation between severity and QoL impairment [14, 21-24, 26-29, 31-37]. Only one study observed that moderate/severe had similar levels of QoL impairment to mild AD patients [15]. However, this study acknowledged that their sample size was small and may have had insufficient power. Furthermore, their assessment of severity was self-rated, and had a different severity distribution from a previous study where severity of a similar sample population was measured based on clinical examination [38].

Beyond severity, there were no other clear links between general QoL impairment and patient demographics, such as age and gender, nor other medical factors, such as presence of concomitant atopic conditions, age at diagnosis, duration of illness and family history of atopy. However, a few studies found correlations between QoL and biological measurements, such as skin hydration $[35,36]$, trans-epidermal 
water loss [36] and several biomarkers [39]. Parent negativity $[31,40]$ was also shown to be associated with a lower QoL. Lastly, steroid phobia, whether from patients [25] or parents [36] was linked to a decreased QoL of AD patients.

\section{Discussion}

QoL was impaired in people with atopic dermatitis in Asian countries with average CDLQI and DLQI scores ranging from 4.8 to 15.2 (CDLQI mean of means $=9.1$ ) and 4.8-12.0 (DLQI mean of means $=9.1$ ) respectively, similar to other studies in Denmark (CDLQI $=8$, DLQI $=5$ ) [4] and the United States (CDLQI $=5.8$, DLQI $=6.6)$ [5]. A review article also quoted that DLQI values of $A D$ patients ranged from 4.5 to 21.4, with the mean of means being 12.2 [41]. While sociocultural differences between these populations may provide some answers as to differences in QoL impairment between countries, it would be hasty to draw major generalizations regarding specific differences between Asian and non-Asian populations, due to the lack of a head to head comparison between the various aspects of QoL affected in these two populations. Different cultures also vary in the way they interpret questionnaires [42], limiting the comparability between countries even with the same QoL instrument.

Aspects of QoL affected were in the domains of symptoms and feelings. In our analysis of the CDLQI, we also found that sleep was particularly affected among children. Indeed $\mathrm{AD}$, like many inflammatory skin conditions such as psoriasis and urticaria, is known to be a highly pruritic disease [43]. This pruritus is enhanced at night, when transepidermal water loss (TEWL) in AD patients is greatest [44], affecting sleep quality through nocturnal awakenings [45]. Thus, clinicians should ensure that these aspects are adequately addressed in the care of their patients.

The nocturnal awakenings by AD patients also disrupted sleep for parents who often engage in co-sleeping (sleeping in the same bed) [46], or when they had to get up to attend to the child, leading to parents having their sleep reduced by a median of 45 and $39 \mathrm{~min} / \mathrm{night}$, respectively [47], consequently impacting family QoL.

There is a clear relationship between severity and degree of QoL impairment. The SCORAD and NESS used in included studies have subjective symptoms like itch as a category in its own right [48]. Itching also affected sleep quality and consequently QoL. Erythema was a feature in SCORAD and EASI [48], and a higher amount of erythema could be linked to greater impact in physical appearance and consequently social embarrassment.

Besides severity, there was no clear link between general QoL impairment and other medical or demographic factors. However, there were only a few studies investigating the effect of each factor on the QoL of AD patients. Therefore, it may be premature to conclude that these factors do not affect QoL. There were also other variables, such as "involvement of visible areas of the body", that have been reported to be determinants of QoL impairment in $\mathrm{AD}$, but were not investigated in the studies we identified.

Our findings highlight the need for clinicians to actively explore the impact of patient's symptoms on QoL and consider using self-reported QoL questionnaires in their routine monitoring $\mathrm{AD}$ patients. This refinement of practice is especially important in the Asian context where patient-doctor communications are traditionally doctor-centered [9, 49], with a focus on symptomatology rather than socio-emotional matters [50].

A limitation of this review is that countries included in this study were not entirely representative of SEA and East Asia; there was a lack of papers from China due to our literature search being confined to English language papers and because of resource constraints not using any Chinese bibliographic databases. Studies from developing countries in Asia were few, and it would be inappropriate to generalize the findings from developed counterparts given the

Table 3 Questions were grouped under headings, and scores for each heading were calculated based on their component questions as specified by the creators of the respective questionnaires

\begin{tabular}{|c|c|c|c|c|c|c|c|c|}
\hline QoL measure & References & $\begin{array}{l}\text { Symptoms } \\
\text { and feelings }\end{array}$ & Leisure & $\begin{array}{l}\text { Personal } \\
\text { relation- } \\
\text { ships }\end{array}$ & School/holidays/work & Treatment & Sleep & Daily activities \\
\hline \multirow[t]{4}{*}{ CDLQI } & Kim DH et al. [26] & $43 \%(1 \mathrm{st})$ & $20 \%$ & $15 \%$ & $27 \%(3 \mathrm{rd})$ & $27 \%$ (3rd) & $33 \%$ (2nd) & NA \\
\hline & Lam et al. [22] & $33 \%(1 \mathrm{st})$ & $17 \%$ & $0 \%$ & $17 \%$ & $33 \%$ (1st) & $33 \%(1 \mathrm{st})$ & NA \\
\hline & Ng et al. [27] & $86 \%$ (2nd) & $94 \%(1 \mathrm{st})$ & $48 \%$ & $27 \%$ & $23 \%$ & $78 \%$ (3rd) & NA \\
\hline & Ghani $[28,29]$ & $43 \%$ (1st) & $19 \%$ & $15 \%$ & $27 \%$ & $37 \%$ (3rd) & $40 \%$ (2nd) & NA \\
\hline \multirow[t]{4}{*}{ DLQI } & Chuh and Chan [14] & $63 \%(1 \mathrm{st})$ & $39 \%$ & $21 \%$ & $27 \%$ & $48 \%$ (2nd) & NA & $40 \%$ (3rd) \\
\hline & Itakura et al. [17] & $42 \%(1 \mathrm{st})$ & $13 \%$ & $7 \%$ & $17 \%(3 \mathrm{rd})$ & $17 \%$ & NA & $22 \%$ (2nd) \\
\hline & Kim et al. [26] & $60 \%(1 \mathrm{st})$ & $38 \%$ (3rd) & $22 \%$ & $53 \%$ (2nd) & $30 \%$ & NA & $37 \%$ \\
\hline & Lam et al. [22] & $50 \%(1 \mathrm{st})$ & $33 \%$ (2nd) & $17 \%$ & $33 \%$ (2nd) & $33 \%$ (2nd) & NA & $33 \%$ (2nd) \\
\hline
\end{tabular}

The scores below are a percentage of the total score for each heading. For each study, the aspects were ranked and indicated in brackets for the top few scores 
differences in health care, illness behaviors and psychosocial characteristics. There remains a need for research to explore the impact of $\mathrm{AD}$ on $\mathrm{QoL}$ in these other countries.

Furthermore, many papers were unclear about the study's inclusion and exclusion criteria, rendering it difficult to assess selection bias (Table 4). Studies should also be transparent about their sampling methods.

The studies included had many differences in their methods, whether in terms of diagnosis or severity scoring or outcome measures. This made comparisons between studies difficult. The interpretation by Ricci et al. [3] was not validated, which may affect the interpretation of DFI scores. Future research should be geared towards the validation of outcome measures and their interpretations and forming a consensus on the instruments used to measure QoL of $\mathrm{AD}$ patients.

\section{Conclusion}

QoL is impaired for both Asian AD sufferers and their family. Sufferers are most affected by the itch, sleep disturbance and embarrassment associated with AD. Severity of disease affects the degree of impairment of QoL on AD patients. Greater attention needs to be given to validation of instruments and consistency of their use, and future research should extend to the investigation of QoL on $\mathrm{AD}$ patients in other developing countries in Asia.

\section{Appendix: instrument in assessing risk of selection and information bias of studies}

\section{Selection bias}

\section{Description of study population}

Inclusion and exclusion criteria should be adequately specific. They should include:

1. Clear definition of atopic dermatitis: papers should state if they used a criteria for diagnosis (e.g. UK Working Party criteria), patient diagnosis or if atopic dermatitis was self reported.

2. Demographics of study population: There should be further inclusion and exclusion criteria on the demographics (e.g. Age) of study population.

3. Setting: Studies should mention the setting in which people with atopic dermatitis were obtained (whether clinical or population based samples).

Categories:
- Clear: All three components (diagnosis, demographics and setting) were specified.

- Unclear: two components were specified.

- No inclusion and/or exclusion criteria: Only one or no component was specified.

\section{Sampling method}

This category assesses if the method of sampling from the study population (as specified by diagnosis, demographics and setting) was truly random and without bias.

Categories:

- Random: the sample was drawn randomly from study population/study specified or that there was complete enumeration from study population.

- Not random: the sample was not drawn randomly. This includes choosing patients based on convenience or any other extrinsic factor.

- Unclear: study did not state how the sample was drawn from study population.

\section{Information bias}

\section{Classification of exposure}

The exposure in this instance refers to the diagnosis of atopic dermatitis. The method in which the diagnosis of atopic dermatitis was established was assessed.

Categories:

- Secure records: study should specify that diagnoses were made by physician or from secured medical records. Physician diagnoses can be established through the use of diagnostic criteria or clinical judgment.

- Self reported: diagnoses of atopic dermatitis were reported by participants of the study, with no verification with secured records.

- Unclear: study did not state how atopic dermatitis was established.

\section{Classification of outcomes}

The outcome in this instance refers to quality of life (QoL) of the study sample. The method of measuring QoL was assessed.

Categories.

- Validated psychometric instruments: instruments which have been evaluated to have adequate validity and reliability. 
Table 4 Risk of bias assessment

\begin{tabular}{|c|c|c|c|c|c|}
\hline References & $\begin{array}{l}\text { Descrip- } \\
\text { tion of study } \\
\text { population }^{\mathrm{a}}\end{array}$ & Sampling method & Follow-up & Classification of exposure & Classification of outcomes \\
\hline Ang et al. [33] & $\mathrm{B}, \mathrm{C}$ & Random & $(-)$ & Secure records & Validated instrument \\
\hline Arima et al. [15] & $\mathrm{A}, \mathrm{B}, \mathrm{C}$ & Random & $(-)$ & Self reported & Validated instrument \\
\hline Aziah et al. [32] & $\mathrm{A}, \mathrm{B}, \mathrm{C}$ & Random & $(+)$ follow up $>80 \%$ & Secure records & Validated instrument \\
\hline Bae et al. [52] & $\mathrm{A}, \mathrm{C}$ & Not random & $(-)$ & Secure records & Validated instrument \\
\hline Chen et al. [30] & $\mathrm{A}, \mathrm{C}$ & Not random & $(-)$ & Secure records & Validated instrument \\
\hline Cheok et al. [16] & $\mathrm{A}, \mathrm{B}, \mathrm{C}$ & Random & $(-)$ & Secure records & Validated instrument \\
\hline Chuh and Chan et al. [14] & $\mathrm{A}, \mathrm{B}, \mathrm{C}$ & Not random & $(-)$ & Secure records & Validated instrument \\
\hline Ghani et al. $[28,29]$ & $\mathrm{A}, \mathrm{B}, \mathrm{C}$ & Random & $(-)$ & Secure records & Validated instrument \\
\hline Higaki et al. [24] & $\mathrm{A}, \mathrm{C}$ & Random & $(+)$ follow-up $>80 \%$ & Secure records & Validated instrument \\
\hline Ho et al. [21] & $\mathrm{A}, \mathrm{B}, \mathrm{C}$ & Random & $(-)$ & Secure records & Validated instrument \\
\hline Hon et al. [53] & $\mathrm{B}, \mathrm{C}$ & Not random & $(-)$ & Secure records & Validated instrument \\
\hline Hon et al. [39] & $\mathrm{A}, \mathrm{B}, \mathrm{C}$ & Random & $(-)$ & Secure records & Validated instrument \\
\hline Hon et al. [54] & $\mathrm{A}, \mathrm{C}$ & Unclear & $(-)$ & Secure records & Validated instrument \\
\hline Hon et al. [36] & $\mathrm{A}, \mathrm{B}, \mathrm{C}$ & Random & $(-)$ & Secure records & Validated instrument \\
\hline Hon et al. [55] & A, B,C & Random & $(-)$ & Secure records & Validated instrument \\
\hline Hon et al. [34] & $\mathrm{A}, \mathrm{B}, \mathrm{C}$ & Unclear & $(-)$ & Secure records & Validated instrument \\
\hline Hon et al. [35] & $\mathrm{A}, \mathrm{B}, \mathrm{C}$ & Unclear & $(-)$ & Secure records & Validated instrument \\
\hline Itakura et al. [17] & $\mathrm{A}, \mathrm{B}, \mathrm{C}$ & Random & $(-)$ & Self reported & Validated instrument \\
\hline Jang et al. [31] & $\mathrm{A}, \mathrm{B}, \mathrm{C}$ & Unclear & $(-)$ & Self reported & Validated instrument \\
\hline Kawashima et al. [25] & $\mathrm{A}, \mathrm{B}, \mathrm{C}$ & Unclear & $(-)$ & Secure records & Validated instrument \\
\hline Kim C et al. [23] & $\mathrm{A}, \mathrm{C}$ & Unclear & Unclear & Secure records & Validated instrument \\
\hline Kim DH et al. [26] & $\mathrm{A}, \mathrm{C}$ & Unclear & $(-)$ & Secure records & Validated instrument \\
\hline $\begin{array}{l}\text { Kwak et al. [18], Lee SH } \\
\text { et al. [19] }\end{array}$ & $\mathrm{A}, \mathrm{C}$ & Random & $(-)$ & Self reported & Validated instrument \\
\hline Lam et al. [22] & $\mathrm{A}, \mathrm{B}, \mathrm{C}$ & Not random & $(-)$ & Secure records & Validated instrument \\
\hline $\mathrm{Ng}$ et al. [27] & $\mathrm{A}, \mathrm{B}, \mathrm{C}$ & Unclear & $(-)$ & Secure records & Validated instrument \\
\hline Oh et al. [40] & A & Unclear & $(-)$ & Unclear & Validated instrument \\
\hline Yano et al. [37] & $\mathrm{C}$ & Unclear & $(-)$ & Secure records & Validated instrument \\
\hline
\end{tabular}

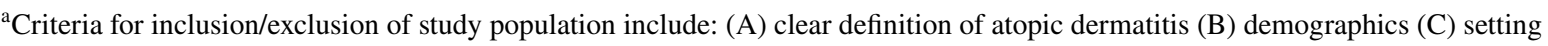

- Non-validated psychometric instruments: instruments which have not been shown to have adequate validity and reliability. This includes translation of validated psychometric instruments which in themselves have not been validated.

- Self-reported QoL: studies in which QoL was asked directly and self-reported.

Acknowledgements The authors would like to thank their colleagues for their valuable input. This research has been previously presented at the European Academy of Allergy and Clinical Immunology (EAACI) Congress 2019 [51].

Author contributions JH, HES, and CA contributed in the study's conception and design. JH and YJC reviewed the studies independently. All authors contributed to the interpretation of results and drafting of manuscript. All authors approved the final manuscript.
Funding Open Access funding enabled and organized by Projekt DEAL. The authors received no specific funding for this work.

\section{Declarations}

Conflict of interest Huang J, Choo YJ, and Smith HE declare no potential conflict of interests. Christian Apfelbacher is a member of the executive committee of the Harmonizing Outcomes Measures for Eczema (HOME) initiative and lead of the quality of life working group within HOME. He has received honorariums and institutional funding from Dr Wolff $\mathrm{GmbH}$, and honorariums from Sanofi Genzyme.

Open Access This article is licensed under a Creative Commons Attribution 4.0 International License, which permits use, sharing, adaptation, distribution and reproduction in any medium or format, as long as you give appropriate credit to the original author(s) and the source, provide a link to the Creative Commons licence, and indicate if changes were made. The images or other third party material in this article are included in the article's Creative Commons licence, unless indicated otherwise in a credit line to the material. If material is not included in 
the article's Creative Commons licence and your intended use is not permitted by statutory regulation or exceeds the permitted use, you will need to obtain permission directly from the copyright holder. To view a copy of this licence, visit http://creativecommons.org/licenses/by/4.0/.

\section{References}

1. Weidinger S, Novak N (2016) Atopic dermatitis. Lancet 387(10023):1109-1122

2. Schmitt J, Spuls P, Boers M et al (2012) Towards global consensus on outcome measures for atopic eczema research: results of the HOME II meeting. Allergy 67(9):1111-1117

3. Ricci G, Bendandi B, Bellini F et al (2007) Atopic dermatitis: quality of life of young Italian children and their families and correlation with severity score. Pediatr Allergy Immunol 18(3):245-249

4. Holm EA, Wulf HC, Stegmann H et al (2006) Life quality assessment among patients with atopic eczema. Br J Dermatol 154(4):719-725

5. Kiebert G, Sorensen SV, Revicki D et al (2002) Atopic dermatitis is associated with a decrement in health-related quality of life. Int J Dermatol 41(3):151-158

6. Torrelo A (2014) Atopic dermatitis in different skin types. What is to know? J Eur Acad Dermatol Venereol 28:2-4

7. Levy RM, Gelfand JM, Yan AC (2003) The epidemiology of atopic dermatitis. Clin Dermatol 21(2):109-115

8. Chen H, Common J, Haines R et al (2011) Wide spectrum of filaggrin-null mutations in atopic dermatitis highlights differences between Singaporean Chinese and European populations. Br J Dermatol 165(1):106-114

9. Matusitz J, Spear J (2015) Doctor-patient communication styles: a comparison between the United States and three Asian countries. J Hum Behav Soc Environ 25(8):871-884

10. Moher D, Liberati A, Tetzlaff J et al (2009) Preferred reporting items for systematic reviews and meta-analyses: the PRISMA statement. Ann Intern Med 151(4):264-269

11. Waters A, Sandhu D, Beattie P et al (2010) Severity stratification of Children's Dermatology Life Quality Index (CDLQI) scores: pa-8. Br J Dermatol 163:121

12. Hongbo Y, Thomas CL, Harrison MA et al (2005) Translating the science of quality of life into practice: what do dermatology life quality index scores mean? J Investig Dermatol 125(4):659-664

13. Dodoo-Schittko F, Brandstetter S, Blecha S et al (2017) Determinants of quality of life and return to work following acute respiratory distress syndrome: a systematic review. Dtsch Arztebl Int 114(7):103

14. Chuh AAT, Chan HHL (2005) Effect on quality of life in patients with pityriasis rosea: is it associated with rash severity? Int J Dermatol 44(5):372-377

15. Arima K, Gupta S, Gadkari A et al (2018) Burden of atopic dermatitis in Japanese adults: analysis of data from the 2013 national health and wellness survey. J Dermatol 45(4):390-396

16. Cheok S, Yee F, Ma JYS et al (2018) Prevalence and descriptive epidemiology of atopic dermatitis and its impact on quality of life in Singapore. Br J Dermatol 178(1):276-277

17. Itakura A, Tani Y, Kaneko $\mathrm{N}$ et al (2018) Impact of chronic urticaria on quality of life and work in Japan: results of a real-world study. J Dermatol 45(8):963-970

18. Kwak Y, Kim Y (2017) Health-related quality of life and mental health of adults with atopic dermatitis. Arch Psychiatr Nurs 31(5):516-521

19. Lee SH, Lee SH, Lee SY et al (2018) Psychological health status and health-related quality of life in adults with atopic dermatitis: a nationwide cross-sectional study in South Korea. Acta Derm Venereol 98(1):89-97

20. Basra M, Gada V, Ungaro S et al (2013) Infants' Dermatitis Quality of Life Index: a decade of experience of validation and clinical application. Br J Dermatol 169(4):760-768

21. Ho RCM, Giam YC, Ng TP et al (2010) The influence of childhood atopic dermatitis on health of mothers, and its impact on Asian families. Pediatr Allergy Immunol 21(3):501-507

22. Lam KM (2010) Quality of life assessment among patients with atopic eczema attending dermatology clinics in Hong Kong. Hong Kong J Dermatol Venereol 18(4):181-188

23. Kim C, Park KY, Ahn S et al (2015) Economic impact of atopic dermatitis in Korean patients. Ann Dermatol 27(3):298-305

24. Higaki Y, Kawamoto K, Kamo T et al (2004) Measurement of the impact of atopic dermatitis on patients' quality of life: a crosssectional and longitudinal questionnaire study using the Japanese version of Skindex-16. J Dermatol 31(12):977-982

25. Kawashima M, QOL Research Forum for Patients with Atopic Dermatitis (2006) Quality of life in patients with atopic dermatitis: impact of tacrolimus ointment. Int J Dermatol 45(6):731-736

26. Kim DH, Li K, Seo SJ et al (2012) Quality of life and disease severity are correlated in patients with atopic dermatitis. J Korean Med Sci 27(11):1327-1332

27. Ng MSY, Tan S, Chan NHQ et al (2018) Effect of atopic dermatitis on quality of life and its psychosocial impact in Asian adolescents. Australas J Dermatol 59(2):E114-E117

28. Ghani AAA, Noor NM, Muhamad R et al (2012) Quality of life and its associated factors among children with atopic eczema in Kelantan, Malaysia. Int J Collab Res Intern Med Public Health 4(11):1816-1827

29. Ghani AAA, Noor NM, Muhamad R et al (2013) Atopic eczema in children: disease severity, quality of life and its impact on family. Int Med J 20(3):320-322

30. Chen YC, Wu CS, Lu YW et al (2013) Atopic dermatitis and non-atopic hand eczema have similar negative impacts on quality of life: implications for clinical significance. Acta Derm Venereol 93(6):749-750

31. Jang HJ, Hwang S, Ahn Y et al (2016) Family quality of life among families of children with atopic dermatitis. Asia Pac Allergy 6(4):213-219

32. Aziah MS, Rosnah T, Mardziah A et al (2002) Childhood atopic dermatitis: a measurement of quality of life and family impact. Med J Malaysia 57(3):329-339

33. Ang SB, Teng CWC, Monika TP et al (2014) Impact of atopic dermatitis on health-related quality of life among infants and children in Singapore: a pilot cross-sectional study. Proc Singap Healthc 23(2):100-107

34. Hon KL, Kung J, Leung T (2018) Evaluating skin equipment for assessing childhood eczema. Australas J Dermatol 59(Supplement 1): 18

35. Hon KL, Kung JSC, Tsang KYC et al (2018) Do we need another symptom score for childhood eczema? J Dermatol Treat 29(5):510-514

36. Hon KL, Tsang YCK, Pong NH et al (2015) Correlations among steroid fear, acceptability, usage frequency, quality of life and disease severity in childhood eczema. J Dermatol Treat 26(5):418-425

37. Yano C, Saeki H, Ishiji T et al (2013) Impact of disease severity on work productivity and activity impairment in Japanese patients with atopic dermatitis. J Dermatol 40(9):736-739

38. Saeki H, Tsunemi Y, Fujita $\mathrm{H}$ et al (2006) Prevalence of atopic dermatitis determined by clinical examination in Japanese adults. J Dermatol 33(11):817-819

39. Hon KLE, Ching GKW, Wong KY et al (2008) A pilot study to explore the usefulness of antibody array in childhood atopic dermatitis. J Natl Med Assoc 100(5):500-504 
40. Oh SH, Bae BG, Park CO et al (2010) Association of stress with symptoms of atopic dermatitis. Acta Derm Venereol 90(6):582-588

41. Lewis V, Finlay AY (2004) 10 years experience of the Dermatology Life Quality Index (DLQI). J Investig Dermatol Symp Proc 9:169-180

42. Lynn P, Japec L, Lyberg L (eds) (2006) What's so special about cross-national surveys? International workshop on comparative survey design and implementation (CSDI). DEU

43. Gupta MA, Gupta AK, Schork NJ et al (1994) Depression modulates pruritus perception: a study of pruritus in psoriasis, atopic dermatitis, and chronic idiopathic urticaria. Psychosom Med 56(1):36-40

44. Lavery MJ, Stull C, Kinney MO et al (2016) Nocturnal pruritus: the battle for a peaceful night's sleep. Int J Mol Sci 17(3):425

45. Gowda S, Goldblum OM, McCall WV et al (2010) Factors affecting sleep quality in patients with psoriasis. J Am Acad Dermatol 63(1):114-123

46. Chamlin SL, Mattson CL, Frieden IJ et al (2005) The price of pruritus: sleep disturbance and cosleeping in atopic dermatitis. Arch Pediatr Adolesc Med 159(8):745-750

47. Moore K, David TJ, Murray CS et al (2006) Effect of childhood eczema and asthma on parental sleep and well-being: a prospective comparative study. Br J Dermatol 154(3):514-518

48. Rehal B, Armstrong A (2011) Health outcome measures in atopic dermatitis: a systematic review of trends in disease severity and quality-of-life instruments 1985-2010. PLoS ONE 6(4):e17520

49. Pun JK, Chan EA, Wang S et al (2018) Health professional-patient communication practices in East Asia: an integrative review of an emerging field of research and practice in Hong Kong, South Korea, Japan, Taiwan, and Mainland China. Patient Educ Couns 101(7):1193-1206

50. Claramita M, Van Dalen J, Van Der Vleuten CP (2011) Doctors in a Southeast Asian country communicate sub-optimally regardless of patients' educational background. Patient Educ Couns 85(3):e169-e174

51. Molloy JD, Clarke A, Morris J, Lim R, Ben-Shoshan M (2019) Abstracts PDS. Allergy 74(S106):130-331

52. Bae JM, Ha B, Lee $\mathrm{H}$ et al (2012) Prevalence of common skin diseases and their associated factors among military personnel in Korea: a cross-sectional study. J Korean Med Sci 27(10):1248-1254

53. Hon KLE, Leung TF, Wong KY et al (2008) Does age or gender influence quality of life in children with atopic dermatitis? Clin Exp Dermatol 33(6):705-709

54. Hon KL, Pong NH, Poon TCW et al (2014) Quality of life and psychosocial issues are important outcome measures in eczema treatment. J Dermatol Treat 26(1):83-89

55. Hon KL, Tsang YC, Poon TC et al (2016) Dairy and nondairy beverage consumption for childhood atopic eczema: what health advice to give? Clin Exp Dermatol 41(2):129-137

Publisher's Note Springer Nature remains neutral with regard to jurisdictional claims in published maps and institutional affiliations. 